\title{
Complete atrioventricular block during tunneled cuffed hemodialysis catheter insertion in a patient with pre-existing left bundle branch block
}

\author{
Eun Woo Choi, Ji Yoon Jung, Jun Huck Su, Sae Huyn Park, Kyu Hyang Cho, Kyung Woo Yoon, \\ Jong Won Park, Jun Young Do, Seok Hui Kang
}

\author{
Division of Nephrology, Department of Internal Medicine, College of Medicine, Yeungnam University, Daegu, Korea
}

Arrhythmias are complications of tunneled cuffed hemodialysis catheter insertion. Most complications associated with arrhythmias occur during guide-wire access, where the guide wire can cause traumatic damage to the conduction system of the heart. Conducting system injury in tunneled cuffed hemodialysis catheter insertion often involves the right bundle, causing right bundle branch block (RBBB). Transient RBBB with sinus rhythm is not usually accompanied by abnormal vital signs. However if patients already have left bundle branch block (LBBB), new onset RBBB can cause complete atrioventricular block (AVB), which can lead to fatal complications requiring invasive treatment. We report on a patient with LBBB who developed complete AVB during hemodialysis catheter insertion.

Keywords: Hemodialysis; Atrioventricular block; Left bundle branch block

\section{INTRODUCTION}

Tunneled cuffed catheters are used to enable adequate vascular access during hemodialysis in patients with end-stage renal disease or acute kidney injury (AKI). Common immediate complications of catheterization include atrial or ventricular tachycardia, pneumothorax, hemothorax, cardiac tamponade, and mediastinal fluid collection [1,2]. However, complete atrioventricular block (AVB) is a rare complication of catheterization.

We report on a patient with pre-existing left bundle branch block (LBBB) who developed complete AVB during tunneled cuffed hemodialysis catheter insertion.

\section{CASE}

A 73-year-old man was admitted with dyspnea. He had

Received: June 30, 2015, Revised: August 11, 2015, Accepted: August 20, 2015

Corresponding Author: Seok Hui Kang, Department of Internal Medicine, College of Medicine, Yeungnam University, 170 Hyeonchung-ro, Namgu, Daegu 42415, Korea Tel: +82-53-680-3844, Fax: +82-53-654-8386

E-mail: kangkang@ynu.ac.kr a history of hypertension, dilated cardiomyopathy, diabetes mellitus, chronic obstructive lung disease, and chronic kidney disease. He was treated with nifedipine. On examination, his blood pressure (BP) and pulse rate were 170/90 $\mathrm{mmHg}$ and 88 beats/min respectively, and he had pitting edema. Chest radiography showed pulmonary congestion and cardiomegaly (Fig. 1). An electrocardiogram (ECG) showed a newly developed LBBB with sinus rhythm (Fig. 2A). Emergency coro-

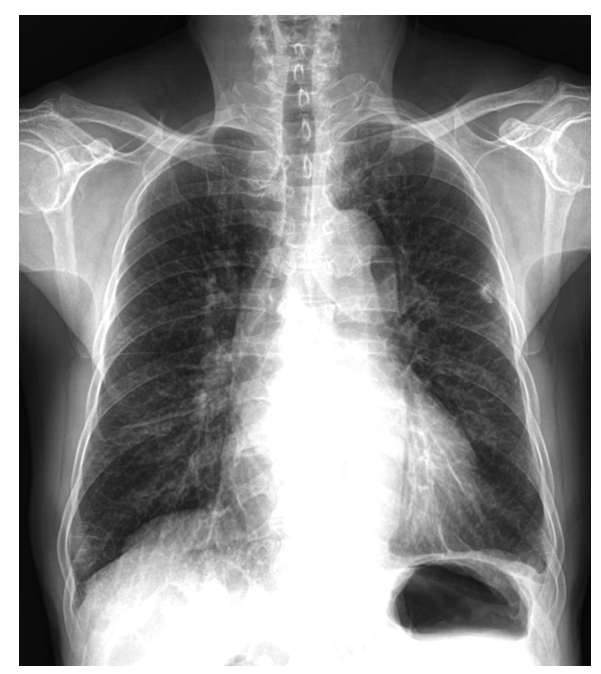

Fig. 1. Chest radiography showed pulmonary congestion and cardiomegaly. 


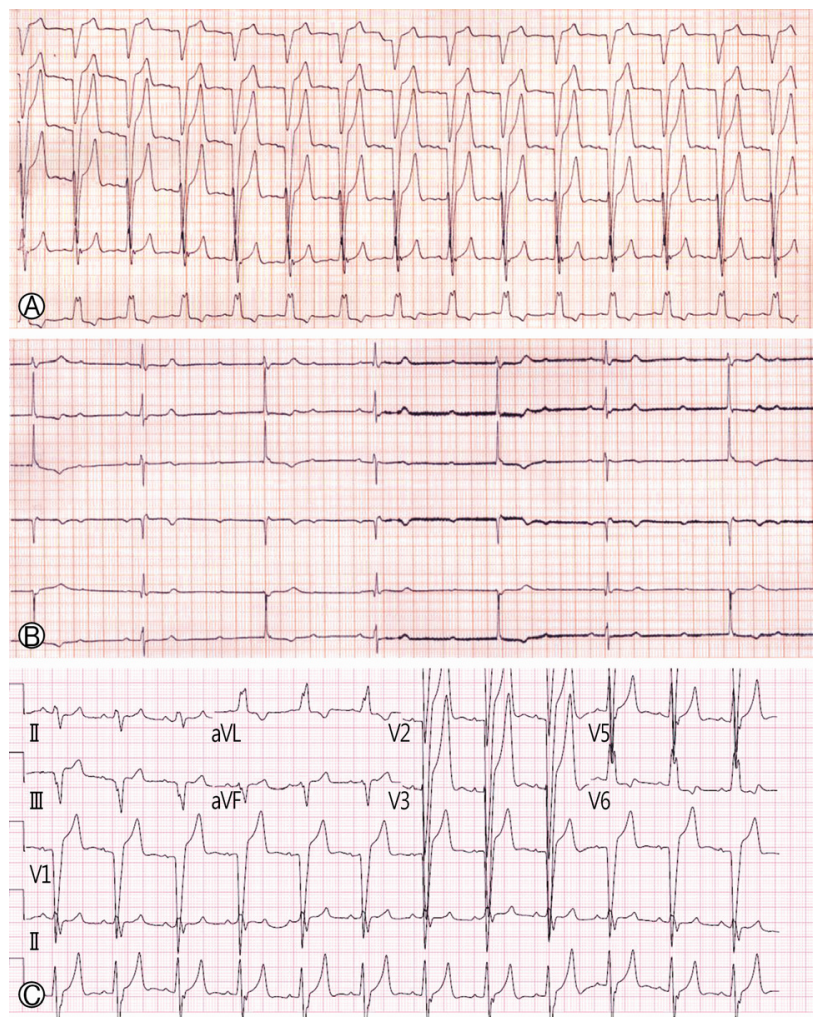

Fig. 2. Electrocardiogram showing pre-existing left bundle branch block (A). Development of complete atrioventricular block during guide-wire insertion (B). Electrocardiogram showing recovered LBBB with sinus rhythm (C).

nary angiography showed no significant coronary artery disease and serum creatinine was $7.17 \mathrm{mg} / \mathrm{dL}$. Thus hemodialysis was required for control of uremia and pulmonary edema.

Tunneled cuff hemodialysis catheter insertion was attempted via the right internal jugular vein. Soon after the guide wire was passed, the ECG trace changed from a LBBB to complete AVB (Fig. 2B). The cardiac monitor showed complete AVB with an escaped ventricular rhythm. BP and ventricular rate were 130/70 $\mathrm{mmHg}$ and 37 beats/min, respectively.

He did not complain of dyspnea or chest discomfort. Cardiac enzymes were in the normal range. The reading was verified using portable echocardiography, and the results remained the same. With close observation, ECG recovered to LBBB without any invasive procedures such as pacemaker or atropine injection (Fig. 2C).

\section{DISCUSSION}

Tunneled cuffed catheters are used to enable adequate vas- cular access during hemodialysis in patients with end-stage renal disease or AKI. The introducer needle is inserted into the right internal jugular vein, and the guide-wire is inserted via a needle. Venous access should be established first, and then maintained. Most complications associated with arrhythmias occur during guide-wire access. Therefore, careful monitoring of ECG, oxygen saturation, and BP is required during catheterization. Common immediate complications of catheterization include atrial or ventricular tachycardia, pneumothorax, hemothorax, cardiac tamponade, and mediastinal fluid collection [1,2]. Atrial or ventricular arrhythmia is a common complication of venous catheterization. The incidence of RBBB during right heart catheterization is approximately $5 \%$, and AVB is very rare in a right heart catheterization [1-3].

The right bundle branch is placed anatomically in the right ventricle endocardium and right bundle branch block (RBBB) can be caused by mechanical trauma induced by the guide-wire [4]. Transient RBBB with sinus rhythm is not usually accompanied by abnormal vital signs. Patients with pre-existent LBBB are at high risk for development of AVB, which can be a life-threatening complication. The patient's vital signs were stable without temporary pacing or inotropics. A patient's vital signs, heart conduction system, duration of catheter placement, and vascular abnormalities must be considered when choosing a hemodialysis catheter. It is generally recommended that the guide-wire length does not exceed $22 \mathrm{~cm}$ and cannulae should be inserted to a depth of $13-16 \mathrm{~cm}$ via the right, and $15-20 \mathrm{~cm}$ via the left jugular vein [5-7]. In addition, guide-wires should be inserted gently and the use of a curved guide-wire may be helpful in reducing mechanical trauma of the heart conduction system.

In summary, hemodialysis catheterization should be performed carefully in patients with pre-existing LBBB with preparation for the possibility of progression to AVB. And, when determining hemodialysis catheter access, the patient's heart conduction system, vital signs, and vascular abnormality should be considered.

\section{REFERENCES}

1. Aydin Z, Gursu M, Uzun S, Karadag S, Tatli E, Sumnu A, et al. Placement of hemodialysis catheters with a technical, functional, and anatomical viewpoint. Int J Nephrol 2012;2012: 302826. 
2. Napalkov P, Felici DM, Chu LK, Jacobs JR, Begelman SM Incidence of catheter-related complications in patients with central venous or hemodialysis catheters: a health care claims database analysis. BMC Cardiovasc Disord 2013;13:86.

3. Sprung CL, Elser B, Schein RM, Marcial EH, Schrager BR. Risk of right bundle-branch block and complete heart block during pulmonary artery catheterization. Crit Care Med 1989; 17:1-3.

4. Munsif AN, Schechter E. Complete block below the His bundle induced by left-sided cardiac catheterization. Cathet Cardiovasc Diagn 1991;24:189-91.
5. Eissa NT, Kvetan V. Guide wire as a cause of complete heart block in patients with preexisting left bundle branch block. Anesthesiology 1990;73:772-4.

6. Seneff M. Central venous catheters. In: Rippe JM, Irwin RS, Fink MP, Cerra FB. Curiey FJ. Heard SO, editors. Procedures and techniques in intensive care medicine. Boston: Little, Brown; 1995. p. 15-36.

7. Gladwin MT, Slonim A, Landucci DL, Gutierrez DC, Cunnion RE. Cannulation of the internal jugular vein: is postprocedural chest radiography always necessary? Crit Care Med 1999;27: 1819-23. 